\title{
Quality of life among patients with chronic renal failure on hemodialysis at the military hospital in southern region of Saudi Arabia
}

Keywords: kidney transplantation, general population, social relationship, quality initiative, psychological state, personal beliefs

Abbreviations: ESRD, end-stage renal disease; KDOQI, kidney disease outcomes quality initiative; QoL, quality of life; ANOVA, analysis of variance; PCS, physical functioning; MCS, mental functioning

\section{Background}

End-stage renal disease (ESRD) is defined as a progressive and irreversible loss in kidney function over a long period (months-years) and sufficiently severe to require maintenance dialysis or kidney transplantation to maintain health of life. ${ }^{1}$ Advances in dialysis treatment have contributed to improved survival of patients with endstage renal disease (ESRD). However, despite improvements in the treatment of ESRD, the level of quality of life $(\mathrm{QoL})$ is much lower for these patients than for the general population. ${ }^{2,3}$

By the end of 2012, there were 12844 patients on dialysis in the Kingdom of Saudi Arabia and expected to rise to 18218 in 2020. There are 182 dialysis centers all over the Kingdom. Of the 12844 patients on hemodialysis, $65 \%$ were treated by $\mathrm{MOH}$ hospitals, $12 \%$ by governmental non- $\mathrm{MOH}$ hospitals and $23 \%$ by private sector. Approximately $54.8 \%$ were males and $88.3 \%$ were Saudis. ${ }^{4}$ Several studies have been conducted on quality of life of CKD patients on hemodialysis; however, there are only few published studies in the Kingdom of Saudi Arabia. We plan to carry this study so as to find about quality of life of chronic kidney disease patients on hemodialysis and to assess their life style and social relationship.

ESRD imposes substantial effects on the patient's QoL by negatively affecting their financial, social, and psychological wellbeing. ${ }^{5}$ The disease also can have impact on patient's overall QoL and other domains like physical, functional, social and mental status. ${ }^{6,7}$ Previous relevant research compared both modalities as well showed that patients undergoing hemodialysis or peritoneal dialysis treatment were found to experience QoL deficits. ${ }^{8}$ According to Kidney Disease Outcomes Quality Initiative (KDOQI) guidelines, there are five stages of CKD based on the glomerular filtration rate. In stage 5 (GFR $<15 \mathrm{ml} / \mathrm{min}$ ), which is defined as end stage renal disease (ESRD) there is total or near-total loss of kidney function which leads to poor life expectancy. ${ }^{9}$ All individuals who reach this stage need renal replacement therapy such as hemodialysis, peritoneal dialysis and renal transplantation as a substitute for the function of their original kidneys. Different renal replacement therapies have different levels of impact on physical, psychological and social health. Quality of life $(\mathrm{QoL})$ as perceived by patients with end stage renal disease is an important measure of patient outcome. WHO has defined QOL as "an individual's perception of their position in life in the context of the culture and value systems in which they live and in relation to their goals, expectations, standards and concerns." It is a broad ranging concept affected in a complex way by the person's physical health,
Volume 6 Issue 5 - 2019

\author{
Norah Ayed Alqahtani,' Metrek Ali Al- \\ Metrek, ${ }^{2}$ Khalid Al-Alsheikh, ${ }^{3}$ Weam Helmy \\ Elnazer $^{4}$ \\ 'Joint Program of Saudi Board in Family Medicine, Khamis \\ Mushait, Saudi Arabia \\ ${ }^{2}$ Academic supervisor, Consultant Family Medicine, Saudi Arabia \\ ${ }^{3}$ Clinical Director of Nephrology Center, Armed Forces \\ Hospitals Southern Region, Saudi Arabia \\ ${ }^{4}$ Adult consultant nephrologist, Armed Forces Hospitals \\ Southern Region, Saudi Arabia
}

Correspondence: Norah Ayed Alqahtani, Joint Program of Saudi Board in Family Medicine, Khamis Mushait, Saudi Arabia, Tel 00966504468402,Email norahayed@hotmail.com

Received: July 28, 2019 | Published: September 17, 2019

psychological state, personal beliefs, social relationships and their relationship to salient features in their environment. ${ }^{10}$

Thus, we need an approach to treat hemodialysis patients involving both nephrologists and psychiatrists as part of a multidisciplinary team to improve their quality of life effectively. ${ }^{11}$ In this study, the QoL is evaluated in hemodialysis patients with reference to their physical, psychological, social, and environmental health dimensions. Also, factors associated with QoL among ESRD patients on hemodialysis are determined. This study investigate the overall and different aspects of quality of life of CKD patients on hemodialysis and to evaluate their lifestyle and social relationship.

\section{Materials and methods}

\section{Subjects}

This cross-sectional study was performed on 164 patients on hemodialysis. The study was conducted at the nephrology center, King Fahd Military hospital, Southern Region in Khamis Mushait city. The ESRD patients included in this study were 20 years and above of both sex and on regular three times per week hemodialysis for at least three months. The study excluded patients with malignancies or multiple organ system failure (liver disease- heart disease- COPD- stroke), and Patients on regular hemodialysis but for less than three months.

Regular hemodialysis for at least three months before patient recruitment in the study was chosen since QoL measurements are less likely to be influenced by metabolic instability and the mode of dialysis treatment after three months of maintenance hemodialysis.

The Researcher fulfilled all the required official approvals. The study proposal was approved by the regional Research and Ethics Committee, King Fahd military hospital Khamis Mushait. Informed consent was asked from all patients and all participants had the right 
not to participate in the study. The researcher explained the purpose to all respondents. Confidentiality and privacy was guaranteed for all participants.

\section{Data collection}

Data collected using a questionnaire composed of two main parts: The first part included patient's demographics (age, gender, nationality, marital status, living status. education, employment status), co-morbid history of sleep disturbance, current smoking, regular exercise, and duration of dialysis. The second part included the Kidney Disease Quality of Life scale (KDQOL-SF-1.3). ${ }^{12}$ Its validated Arabic version was utilized. ${ }^{13}$ The KDQoL scale is disease-targeted and focuses on particular health-related concerns of individuals with kidney disease, patients on dialysis, and the effects of the kidney disease on daily life, the burden of kidney disease, work status, cognitive function, and quality of social interaction, sexual function, social support, dialysis staff encouragement, and patient satisfaction. Its 36 items are categorized into six domains: general health, physical, emotional, social status, illness impact, and financial and medical satisfaction. ${ }^{14}$ The scoring of the tool responses was done according to the guidelines of the KDQOL-SF 12.By using Epi-info software, version 7, putting into consideration that the expected proportion of outcome is $50 \%$ as rate of poor quality of life among hemodialysis patients, the worst accepted proportion is $\pm 5 \%$ and the level of confidence is $95 \%$, sample size was estimated to be 164 . This sample size constituted almost $56 \%$ of the patients registered in the nephrology center, King Fahd military hospital, Khamis Mushait.

\section{Statistical analysis}

The data was collected and verified by hand then coded before computerized data entry. The Statistical Package for Social Sciences (SPSS) software version 22.0 used for data entry and analysis. Descriptive statistics (e.g. number, percentage, mean, range, standard deviation) and analytic statistics using chi-square " $\chi 2$ " will be applied. The one-way analysis of variance (ANOVA) or Kruskall-Wallis tests for skewed data was utilized to compare continuous variables between more than two independent groups while the Student's t-test or the Mann-Whitney test for skewed data was applied for comparisons between two groups. Pearson correlation was used to assess the relationship between quality of life and continuous variables (e.g., age, duration). Following univariate analysis, all demographic and clinical variables with $\mathrm{P} \leq 0.05$ were entered as predictor variables in multiple regression models. P-values $\leq 0.05$ was considered as statistically significant.

\section{Results}

The present study was conducted on 164 patients, $84(51.2 \%)$ of them were males whereas $80(48.8 \%)$ were females, a questionnaire of 45 questions to investigate quality of life of patients and demographics. There were 52(31.7\%) filled the survey alone, 60(36.6\%), 44(26.8\%) and $8(4.9 \%)$ needed help of a family member or a friend, a physician or other health care provider and someone else respectively. The age range of participants was 20-93 years old with a mean of $58 \pm 17$. There were $119(72.6 \%)$ were married while $45(27.4 \%)$ weren't.

Most of patients were living with others 157(95.7\%) and weren't working $151(92.1 \%)$ while only $7(4.3 \%)$ were living alone and $13(7.9 \%)$ were working. The annual income for large majority of participants $49(29.9 \%)$ was less than 20.000 Saudi Riyal (SR), 19(11.6\%),11(6.7\%), 6(3.7\%), 9(5.5\%) and 6(3.7\%) their annual salaries were 20.000-40.000 SR, 40.000-80.000 SR, 80.000-150.000SR, 150.000-300.00SR and more than 300.00 SR respectively, while $64(39 \%)$ said that they didn't know. There were $163(99.4 \%)$ Saudi individuals, whereas $1(0.6 \%)$ only was African or black American. Large number of participants 140(85.4\%) did not smoke, while 20(12.2\%) were ex-smokers and 4(2.4) were smoking. Around half of patients $77(47 \%)$ had diabetes and the majority had hypertension $118(72.4 \%)$, Close percent were found to suffer from sleep complaint $(48.8 \%)$ and the rest $(51.2 \%)$ didn't suffer from sleep complaint. there were $127(77.4 \%$ ) didn't exercise daily, while $37(22.6 \%)$ performed physical activities; Table 1 summarizes the characteristics of patients. The range of duration of blood dialysis was 5-264 months with a mean of $52 \pm 50$.

Table I Characteristics of patients

\begin{tabular}{|c|c|c|}
\hline Characteristics & $\mathbf{N}$ & $\%$ \\
\hline \multicolumn{3}{|l|}{ Sex } \\
\hline Male & 84 & 51.2 \\
\hline Female & 80 & 48.8 \\
\hline \multicolumn{3}{|l|}{ Filling the survey } \\
\hline Alone & 52 & 31.7 \\
\hline Need help of family member & 60 & 36.6 \\
\hline Need help of physician & 44 & 26.8 \\
\hline Need help of someone else & 8 & 9.4 \\
\hline \multicolumn{3}{|l|}{ Marital status } \\
\hline Married & 119 & 72.6 \\
\hline Not married & 45 & 27.4 \\
\hline \multicolumn{3}{|l|}{ Living } \\
\hline With others & 157 & 95.7 \\
\hline Alone & 7 & 4.3 \\
\hline \multicolumn{3}{|l|}{ Working } \\
\hline Yes & 13 & 7.9 \\
\hline No & $15 \mid$ & 92.1 \\
\hline \multicolumn{3}{|l|}{ Annual income } \\
\hline less than $20.000 \mathrm{SR}$ & 49 & 29.9 \\
\hline $20.000-40.000 \mathrm{SR}$ & 19 & 11.6 \\
\hline $40.000-80.000 \mathrm{SR}$ & II & 6.7 \\
\hline $80.000-150.000 S R$ & 6 & 3.7 \\
\hline $150.000-300.00 S R$ & 9 & 5.5 \\
\hline more than 300.00 SR & 6 & 3.7 \\
\hline I don't know & 64 & 39 \\
\hline \multicolumn{3}{|l|}{ Nationality } \\
\hline Saudi & 163 & 99.4 \\
\hline African & I & 0.6 \\
\hline \multicolumn{3}{|l|}{ Smoking } \\
\hline Yes & 4 & 2.4 \\
\hline No & 140 & 85.4 \\
\hline Ex-smoker & 20 & 12.2 \\
\hline
\end{tabular}


Table Continues...

\begin{tabular}{lll}
\hline Characteristics & N & $\%$ \\
\hline Diseases & 77 & 47 \\
Diabetes & 118 & 72.4 \\
Hypertension & & \\
Sleep complaint & 80 & 48.8 \\
Yes & 84 & 51.2 \\
No & & \\
Physical activity & 37 & 22.6 \\
Yes & 127 & 77.4 \\
No & & \\
\hline
\end{tabular}

There was a question (Q22) to investigate the overall health of patients, it was found that there was a weak correlation between overall health of patients and the sleep of patients $(\mathrm{r}=0.471, \mathrm{P}$-value $<0.001)$, a weak negative correlation existed between overall health of patients and age $(\mathrm{r}=-0.159, \mathrm{P}$-value $=0.042)$ and there was a weak negative correlation existed between overall health of patients and duration of dialysis $(r=-0.159, \mathrm{P}$ value $=0.042)$.

There was a significant difference between overall health of patients and health state of patients in the last 30 days ( $\mathrm{P}$-value $=0.009)$, also there was a significance was found regarding overall health status of patients and living alone or with someone (P-value $=0.006)$. Regarding overall health of patients, there was a significant difference between working patients and those who didn't work (P-value $=0.02)$, also there was a significant difference in overall feeling health for patients regarding suffering from sleep complaint $(\mathrm{P}-\mathrm{value}=0.054)$ and regarding performing physical activity daily $(\mathrm{P}$-value $<0.001)$.

The QOL investigated by KDQOL questionnaire depending on 5 subscales; symptoms and problems, effects of the kidney disease, burden of the kidney disease, physical functioning and mental functioning. The percents of patients scored less than 50 in each sub scale are shown in Figure 1.The mean of symptoms and problems (S) was 74 with a range of 12.5-100, whereas that of effects of the kidney disease (E) was 56.5 for mean and $0-100$ for range, 31.5 and $0-100$ for mean and range of burden of the kidney disease (C), 37.4 and 12.32-64 for mean and range of SF-12 physical functioning (PCS) and 43.5 and 15.8-67.64 for mean and range of SF-12 mental functioning (MCS).

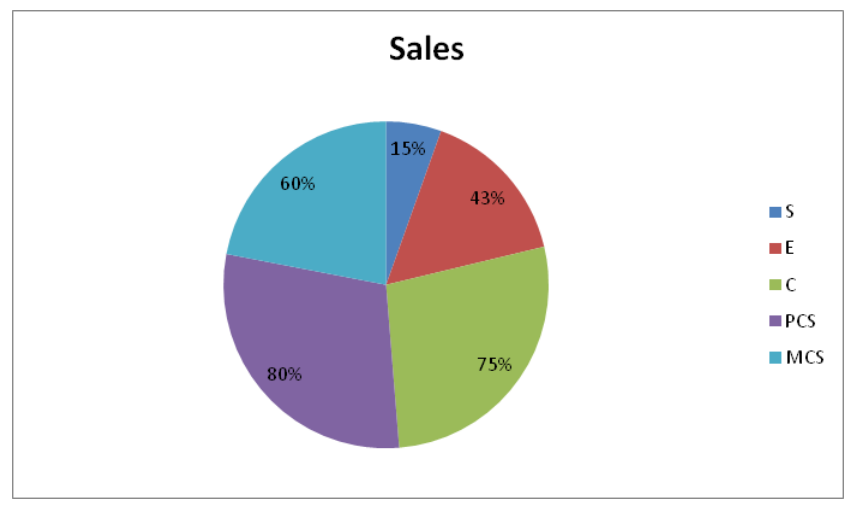

Figure I Quality of life subscales and percent of patients.

\section{Discussion}

The present study, included 164 patients with similar percent for both groups, male $51.2 \%$ and female $48.8 \%$. Regarding overall health of patients, there was a weak positive correlation between overall health of patients and sleep, the overall health and quality of life of patients increase by increasing hours of sleep, those who sleep inadequate hours suffer less quality of life.

There was a weak negative correlation between Qol of patients and age, this means that overall health of patients decreases with increasing age and hence decrease in quality of life. This was in agreement with a study by Guerra-Guerrero et al. ${ }^{15}$ who reported a negative correlation between age and all subscales assessed, except in the effects of the Kidney disease.

Also, in this study a weak negative correlation was found between overall health of patients and duration of dialysis $(r=-0.159)$, this indicated that increasing the duration of dialysis results in decrease overall health and overall quality of life, so early diagnosis is very important to avoid the need for long duration of dialysis. In a Saudi study, ${ }^{16}$ it was found that there was a negative association between QoL and the duration of dialysis, the same findings were reported by Ginieri-Coccossis et al. ${ }^{17}$ who revealed that demonstrated decrease in QoL in patients who have been on dialysis for long time. Our findings supports the previous findings, whereas the opposite findings were demonstrated by another study, ${ }^{18}$ where it was found that there was no change in QoL over time, there was even improvement in QoL over time. However, this opposite results can be attributed to other study design, other inclusion and exclusion criteria and shorter duration of study.

The current study showed that there was a significant difference $(\mathrm{P}-$ value $=0.006$ ) between overall health of patients and living alone or with other, most of patients lived with others, it was indicated that living alone was an independent predictor for the mental health component improvements overtime. ${ }^{19}$ There was a significant difference $(\mathrm{P}$-value $=0.02)$ in overall health of patients regarding working. Exercise affects overall health of patients and quality of life, there was a significant difference in overall health of patients and quality of life regarding performing exercise daily ( $\mathrm{P}$-value $<0.001$ ), the daily exercise has good impact in quality of life of patients. Regarding Qol, the reference value was 50 points, there were high percentage of patients in three categories scored less than 50 points.

The KDQOL questionnaire that was used for investigating QOL depended on 5 subscales including burden of the kidney disease. Regarding burden of kidney disease, there were $75 \%$ of patients had scores less than 50 points, according to physical functioning there were $80 \%$ of patients scored less than 50 points and $60 \%$ of patients scored less than 50 points regarding mental functioning. This agreed with a previous study, ${ }^{15}$ where there were $76 \%$ of patients scored less than 50 points regarding burden of disease and regarding both physical and mental functioning subscales more than $50 \%$ scored less than 50 points for each subscale. Shrestha et al. ${ }^{20}$ demonstrated that physical health was the most severely affected domain of KDQOL, where the mean score was 33.36, this is close to our findings where the mean score for physical functioning was 37.4 , however the mean score of burden of kidney disease was lower 31.5 than that of physical functioning, this indicates that burden of kidney disease was the most affected domain.

The mean score of physical functioning of our study was lower than that of a previous Saudi study, ${ }^{16}$ where it was reported that the mean score of physical functioning was 53.1. The mean score of physical functioning was less than that of mental functioning, this agreed with previous other studies. ${ }^{15,21-23}$ These findings showed that the ability of patients to physically adapt to their situation over the time. The 
scored mean score of physical functioning (37.4) was lower than that of mental functioning (43.5), this was in agree mental with other previous studies. ${ }^{21-23}$ This can be explained by the ability of patients to psychologically adapt over time with their situation. In the present study regarding symptoms and problems, low percent of participants scored less than 50 points, a similar result was reported in a previous study, ${ }^{15}$ regarding symptoms and problems subscale. The current study revealed that less than half of participants scored less than 50 points regarding effects of kidney diseases, the same was reported by Guerra-Guerrero et al. ${ }^{15}$ This similarity in results can be attributed to the use of the same questionnaire and similar study design.

\section{Recommendations}

Further observational and interventional studies regarding improving quality of life are required for better patient-centered care.

\section{Conclusion}

Quality of life was affected by several factors including sleep hours, age, duration of dialysis, performing exercise and living alone or with someone. Quality of life decreased regarding three parameters; Burden of the Kidney disease, Physical Functioning and Mental Functioning.

\section{Funding and granting}

This research received no specific grant from any funding agency in the public, commercial or not-for-profit sectors.

\section{Contributorship statement}

Main author did the proposal writing and data analysis contracting and follow up, while the second author did validation for the tool and administrative follow up and did final manuscript writing and submission. Both author did for data collection procedure.

\section{Acknowledgments}

We thank Administration of Armed Forces Hospital Southern Region for assistance with facilitating the data collection.

\section{Competing interests}

The authors have no competing interests to declare.

\section{References}

1. Müller H, Gwinner W, Haltenhof $\mathrm{H}$, et al. Psychological risk factors of kidney transplant patients. Kidney Int. 2010;77(9):833.

2. Valderrabano F, Jofre R, Lopez-Gomez JM. Quality of life in end-stage renal disease patients. Am J Kidney Dis. 2001;38(3):443-464.

3. Finkelstein FO, Finkelstein SH. Depression in chronic dialysis patients: assessment and treatment. Nephrol Dial Transplant. 2000;15(12):19111913.

4. Annual report. Saudi Center for Organ Transplantation. 2013.

5. Christensen A, Ehlers S. Psychological factors in end-stage renal disease: an emerging context for behavioral medicine research. $J$ Consult Clin Psychol. 2002;70(3):712-724.
6. Suet-Chaing WL. The quality of life for Hong Kong dialysis patients. $J$ Adv Nurse. 2001;35(2):218-227.

7. Bakewell AB, Higgins RM, Edmunds ME. Quality of life in peritoneal dialysis patients: Decline over time and association with clinical outcomes. Kidney Int. 2002;61(1):239-248.

8. Griva K, Newman S. Quality of life in end-stage renal disease and treatments. In: Anagnostopoulos F, Karademas E, editors. Special Issues in Health Psychology. A Greek Perspective. Athens: Livani; 2007.

9. National Kidney Foundation. K/DOQI clinical practice guidelines for chronic kidney disease. 2002.

10. De Vries J, Van Heck GL. The World Health Organization Quality of Life Assessment Instrument (WHOQOL-100): Validation Study with the Dutch Version. European Journal of Psychological Assessment. 1997;13(3):164 178.

11. Ruiz de Gauna R, Minguela Pesquera I, Ocharán-Corcuera J, et al. The social environment of patients on peritoneal dialysis. Nefrologia. 2008;28 Suppl 6:133-136.

12. Hays RD, Kallich JD, Mapes DL, et al. Kidney Disease Quality of Life Short form (KDQoL- SF TM), Version 1.3. A Manual for Use and Scoring. Santa Monica CA: RAND; 1997.

13. AbdElHafeez S, Sallam SA, Gad ZM, et al. Cultural adaptation and validation of the "Kidney Disease and Quality of Life - Short Form (KDQOL-SFTM) version 1.3" questionnaire in Egypt. BMC Nephrol. 2012;13:170.

14. Al Wakeel JA, Bayoumi M, Al Suwaida A, et al. Influences on quality of life in peritoneal dialysis patients. The renal society of Australasia journal. 2009;5(3):127-132.

15. Guerra-Guerrero V, Sanhueza-Alvarado O, Caceres-Espina M Quality of life in people with chronic hemodialysis: association with sociodemographic, medical-clinical and laboratory variables. Rev Latino Am Enfermagem. 2012:20(5):838-846.

16. Bayoumi M, Al harbi A, Al Suwaida A, et al. Predictors of Quality of Life in Hemodialysis Patients. Saudi J Kidney Dis Transpl. 2013:24(2):254 259 .

17. Ginieri-Coccossis M, Theofilou P, Synodinou C, et al. Quality of life, mental health and health beliefs in hemodialysis and peritoneal dialysis patients: Investigating differences in early and later years of currents treatment. BMC Nephrol. 2008;9:14.

18. Harries SA, Lamping DL, Brown EA, et al. Clinical outcomes and quality of life in elderly patients on peritoneal dialysis versus hemodialysis. Perit Dial Int. 2002:22(4):463-470.

19. Morsch C, Goncalves L, Barros E. Health-related quality of life among haemodialysis patients-relationship with clinical indicators, morbidity and mortality. J Clin Nurs. 2006;15(4):498-504.

20. Shrestha S, Ghotekar LR, Sharma SK, et al. Assessment of quality of life of end stage renal disease on different modalities of treatment. JNMA $J$ Nepal Med Assoc. 2008;47(169):1-6.

21. Bohlke M, Leite D, Scaglioni S, et al. Predictors of quality of life among patients on dialysis in southern Brazil. Sao Paulo Med J. 2008;126(5):252256.

22. http://www.redalyc.org/pdf/647/64750305.pdf

23. https://dialnet.unirioja.es/servlet/libro? codigo $=698090$ 